Cahiers « Mondes anciens »

ANCIENS

Histoire et anthropologie des mondes anciens

$4 \mid 2013$

Journées doctorales ANHIMA 2010 et 2011

\title{
Conclusion
}

Autour de l'image : méthodes et problèmes

\section{Sylvia Estienne}

\section{(2) OpenEdition}

Journals

Édition électronique

URL : http://journals.openedition.org/mondesanciens/1095

DOI : 10.4000/mondesanciens. 1095

ISSN : 2107-0199

Éditeur

UMR 8210 Anthropologie et Histoire des Mondes Antiques

Référence électronique

Sylvia Estienne, «Conclusion », Cahiers « Mondes anciens » [En ligne], 4 | 2013, mis en ligne le 01 juillet 2013, consulté le 03 mai 2019. URL : http://journals.openedition.org/mondesanciens/1095; DOI :

10.4000/mondesanciens.1095

Ce document a été généré automatiquement le 3 mai 2019.

\section{(c) $($ i) $(9)$}

Les Cahiers «Mondes Anciens " sont mis à disposition selon les termes de la licence Creative Commons Attribution - Pas d'Utilisation Commerciale - Pas de Modification 4.0 International. 


\title{
Conclusion
}

\author{
Autour de l'image : méthodes et problèmes
}

\author{
Sylvia Estienne
}

1 Depuis les années 1990, qui ont vu la multiplication des ouvrages consacrés à l'utilisation des images par les historiens, on aurait pu croire réglées certaines des questions posées par l'analyse des documents iconographiques. Certes, nul ne conteste plus que les images soient une source à part entière; mais au-delà des discours de la méthode, faire une histoire des images, ou plutôt une histoire par l'image, reste périlleux. Si quelques études pionnières ont ouvert la voie, les enquêtes menées sont encore très parcellaires. Cette rencontre montre que les jeunes historiens de l'Antiquité, armés d'outils épistémologiques et bibliographiques récents, n'en sont pas moins confrontés à des problèmes de méthode et d'interprétation, quand ils abordent ce type de documentation. Les quatre interventions se sont toutes révélées riches et foisonnantes; partant du thème commun de l'image, elles frappent d'abord par la diversité de leurs approches. Les différences tiennent certes à la nature des images étudiées, à leur place dans l'enquête, aux questionnements auxquels elles sont soumises. Au-delà de la diversité des images analysées et des démarches adoptées, on peut toutefois dégager quelques-unes des difficultés communément rencontrées. C'est donc aux aspects méthodologiques que cette journée nous invite à réfléchir. Les réponses apportées par chacun des intervenants révèlent la complexité des enjeux interprétatifs, mais aussi la conscience claire des méthodologies à mettre en œuvre et des limites imposées à l'analyse.

\section{Répertoires, corpus, mise en série}

2 La première exigence scientifique à laquelle se heurte l'historien est celle de la sélection de la documentation et de la constitution d'un corpus pertinent. Qu'il s'agisse de peinture sur céramique ou de représentations monétaires, les quatre communications entendues aujourd'hui portent sur des images qui se prêtent toutes a priori à une analyse sérielle. Cependant les enjeux méthodologiques de ce type d'analyse varient d'une communication à l'autre. 
3 Les images peintes sur céramique, au cœur de trois des exposés, offrent l'avantage d'un matériau largement inventorié et répertorié ${ }^{1}$. Forts de la cohérence des répertoires existants, les intervenants ont pu élaborer leur propre corpus thématique, à partir d'un ensemble homogène que ce soit par la nature des supports, le contexte de production ou le référent culturel. Les choix qui président à la sélection de chacun sont toutefois assez différents.

4 Si V.Zachari peut résolument centrer sa recherche sur une composition figurative particulière tout en balayant l'ensemble de la production attique classique, c'est que le terrain sur lequel elle s'avance est en partie défriché; forte d'une connaissance approfondie de l'imagerie attique entre le $\mathrm{VI}^{\mathrm{e}}$ et le $\mathrm{IV}^{\mathrm{e}} \mathrm{s}$., rendue possible notamment par l'existence d'inventaires généraux ${ }^{2}$, elle étudie l'association de l'autel et du pilier hermaïque dans la représentation de l'espace. Elle s'appuie également sur les résultats d'études antérieures, comme celles de G. Ekroth ou D. Jaillard ${ }^{3}$. Elle peut ainsi dégager finement les logiques propres à la représentation de l'espace sacré dans l'imagerie attique.

5 Les recherches de $\mathrm{M}$. Baggio portent sur un autre répertoire, celui des vases peints de Grande-Grèce, pour lesquels elle peut s'appuyer sur les travaux d'A. D. Trendall, de M. Denoyelle et M. Iozzo ${ }^{4}$. À l'inverse de V. Zachari, elle a choisi de centrer son enquête sur une production très restreinte, celle des loutrophores du peintre de Baltimore, soit un corpus a priori assez réduit (huit vases). Cette sélection, justifiée par des critères à la fois chronologiques et fonctionnels, est toutefois mise en perspective par la comparaison avec d'autres productions similaires, en termes de typologie ou de chronologie. Ces deux intervenantes ont donc choisi des stratégies différentes pour aborder une même question, celle des mécanismes figuratifs à l'œuvre dans une culture donnée, celle de l'Athènes classique d'une part, celle de la Grande-Grèce hellénistique d'autre part.

C'est en partant d'un point de départ un peu différent que G. Cogan affronte des problématiques proches. Ses recherches sur les récompenses agonistiques dans la culture grecque l'ont naturellement amenée à s'intéresser au répertoire céramique attique, mais la documentation qu'elle a sélectionnée, celle des scènes où figurent des hydries comme objets de prix, l'invite à dépasser ses propres problématiques et à tenir compte d'un corpus plus large, qui inclut des scènes de femmes à la fontaine ou des représentations homériques. La logique propre au corpus l'emporte alors sur les présupposés de la recherche et ouvre de nouvelles voies à l'analyse.

7 Le matériel numismatique étudié par Y. Berthelet pourrait donner lieu à des analyses similaires: là encore il s'agit d'un répertoire bien inventorié, daté et porteur d'un système figuratif délimité. La stratégie adoptée est toutefois très différente; en sélectionnant des représentations d'instruments sacerdotaux, l'auteur s'intéresse moins à la logique figurative de ce répertoire, assez banal il est vrai, qu'à la cohérence d'un système symbolique. Significativement, il utilise autant les apports du répertoire numismatique ${ }^{5}$ que ceux du répertoire prosopographique ${ }^{6}$. Ce faisant, il offre un contrepoint ponctuel, mais stimulant, à une démarche iconographique plus classique, fondée sur une approche typologique ${ }^{7}$.

8 Les différentes approches développées par ces quatre intervenants s'appuient donc sur la sérialité et l'homogénéité de répertoires préexistants. Cela leur permet de repérer plus vite les écarts ou les normes, d'affiner l'appréhension chronologique de leur objet 
d'étude, de mettre en perspective leurs résultats, sans pour autant les dispenser d'une nécessaire sélection.

\section{Images-objets}

9 À la diversité des images étudiées répond la multiplicité des approches, iconographique pour les uns, historique pour les autres. Les différentes communications ont néanmoins en commun de travailler sur des productions qui ne sont pas données comme artistiques. À ce titre, il faut sans doute tenir compte du filtre imposé à l'imagerie attique par notre propre réception, qui tend à isoler les scènes de leur support et à privilégier les détails. Il ne s'agit pas d'en nier la dimension esthétique, mais de souligner que la lecture est déterminée par la nature du support. S'il est évident pour tous que l'image est indissociable de l'objet qui la porte, les rapports entre support et figuration ont été diversement pris en compte et analysés.

Dans le cas des scènes peintes sur céramique, le point focal a été nettement mis sur la figuration: l'attention des intervenants a porté principalement sur la composition des scènes, les relations entre les différents éléments représentés. Le lien de l'image avec son support, le vase, n'a pas pour autant été occulté. V. Zachari a ainsi souligné la façon dont les peintres utilisaient la forme même du vase pour jouer sur certains effets visuels ; celleci impose en effet un cadre contraignant, dont les peintres peuvent jouer pour donner, paradoxalement, l'illusion d'un espace ouvert, voire dynamique.

11 De son côté, G. Cogan a été particulièrement attentive au jeu d'échos créé par la représentation d'hydries comme symboles de la victoire sur des hydries, elles-mêmes prix pour la victoire. Les hydries représentent ainsi un peu moins de la moitié des vases qu'elle étudie en détail. Le jeu devient plus complexe quand on prend en compte d'autres vases utilisés comme récompenses en contexte agonistique, comme les amphores panathénaïques. Le réseau dense d'interrelations que tisse G. Cogan dans ses analyses déploie progressivement toute la polysémie de l'hydrie, bien au-delà d'une simple mise en abyme; plus qu'un simple objet de prestige, l'hydrie acquiert alors dans l'image une place spécifique, symbolisant le passage, la transition d'un état à un autre.

Elle rejoint en cela les prémisses de l'étude de M. Baggio, qui s'appuie notamment sur la distinction empruntée à J.-M. Moret entre " valeur dénotative " et " valeur connotative " des objets dans les productions apuliennes ${ }^{8}$. C'est à dessein que M. Baggio a choisi de restreindre son étude à un seul type de vase, des loutrophores, objets de prestige liés au monde féminin. Ce choix se justifie autant par la cohérence du système figuratif que par l'importance accrue de ces vases dans le contexte funéraire apulien à partir de la fin du IV es. av. J.-C. À travers cette production, ce sont les mécanismes de valorisation de l'identité féminine qui sont mis en lumière.

13 Les représentations monétaires étudiées par Y. Berthelet semblent en revanche envisagées davantage comme des objets que comme des images. L'identité du magistratmonnayeur, la chronologie des émissions, les conditions de leur diffusion ont été les facteurs privilégiés dans la démonstration pour rendre compte du sens à donner à ces images. Sans doute n'est-ce pas un hasard si les monnaies les plus discutées ont été frappées dans le contexte troublé des guerres civiles, que ce soit les émissions de Sylla ou celles du triumvirat: la fonction de légitimation est redoublée par la multiplication des 
ateliers monétaires et les besoins en numéraire. La fonction politique de l'objet l'emporte ici nettement sur sa valeur iconographique.

\section{Objets dans l'image}

14 Toutes les communications ont en commun de centrer l'analyse sur un ou des objets précis dans le champ de l'image. Ces choix sont significatifs, même s'ils ne s'inscrivent pas toujours dans des démarches similaires : si V. Zachari ou M. Baggio partent d'une logique interne à leur répertoire respectif (fréquence ou apparition d'une association d'objets), G. Cogan et $Y$. Berthelet isolent un objet significatif pour leur thème de recherche (hydrie comme objet de prix ; cruche comme instrument cultuel) pour en dégager la valeur dans un système de représentation donné. Tous néanmoins réfléchissent à leur manière aux relations entre objet réel et objet représenté, valeur fonctionnelle et valeur symbolique.

V. Zachari note ainsi qu'il y a une grande similarité entre les autels réels et ceux figurés dans l'imagerie attique; si leur association avec des piliers hermaïques est bien attestée dans le répertoire céramique, il n'y a en revanche aucune attestation archéologique de cette association dans les espaces réels. En tant qu'objets, ils se veulent une représentation fidèle, mais en tant que marqueurs dans l'image, ils participent à la représentation d'un espace idéal.

16 Sur les loutrophores du peintre de Baltimore, M. Baggio met en évidence un motif relativement inhabituel d'objets, exposés en marge du champ de l'image et déconnectés de toute manipulation: ciste, alabastron ou kalathos ainsi représentés hors de tout contexte fonctionnel acquièrent une valeur symbolique forte, liée au statut du/de la défunt(e). En élargissant le champ de son enquête, elle montre comment des objets du quotidien peuvent être « refonctionnalisés » dans l'image comme signe du statut et indice de prestige social.

17 G. Cogan procède à une recontextualisation minutieuse des hydries dans l'imagerie attique. Réduire celles-ci à leur simple valeur fonctionnelle (transport et conservation de l'eau) ne lui paraît guère satisfaisant et tendrait à mettre en avant l'hétérogénéité des scènes où ces objets apparaissent : scènes de victoire athlétique, femmes à la fontaine, scènes mythologiques. C'est donc à partir de la valeur symbolique de l'objet qu'elle cherche à dégager la fonction dans l'image : elle y voit un signe du passage d'un statut à un autre.

De son côté Y. Berthelet définit rapidement la forme et l'usage de la cruche que l'on appelle conventionnellement urceus, voire guttus; mais ce n'est qu'une fois démontré le lien, dans la documentation numismatique, entre cette cruche, accompagnée de la crosse augurale (lituus), et l'appartenance du magistrat-monnayeur au collège des augures qu'il s'interroge sur l'utilisation cultuelle de ce vase et donc sa pertinence dans le système de représentation symbolique des sacerdoces romains. Dans d'autres contextes, sur les autels par exemple, la cruche est communément associée aux instruments du sacrifice, rituel pourtant peu attesté dans les obligations cultuelles de ce collège. La cohérence du système figuratif numismatique doit donc être mise à l'épreuve du reste de la documentation.

Chacune des contributions montre donc que l'analyse des objets dans l'image doit pour se faire être soigneusement contextualisée ; l'objet, d'abord isolé par le regard porté sur lui, 
rentre alors dans un système de signes ${ }^{9}$, propre aussi bien aux pratiques figuratives qu'au système de valeurs d'une société donnée.

\section{Texte et image}

Plusieurs des contributions permettent enfin d'aborder les relations entre image et texte dans toute leur complexité, même si cet aspect a été peu développé par les intervenants. Force est de reconnaître que la part du texte dans l'image est très inégale dans les différents cas envisagés : réduit, voire absent des peintures sur vase, omniprésent, mais souvent transparent sur les monnaies. Le recours à l'écriture constitue un surcroît de sens dans le premiers cas. Indispensable sur les monnaies, le texte écrit n'a pas pour autant la fonction d'expliciter l'image; images et légendes monétaires sont a priori autonomes, mais indissociables pour la compréhension. Les liens entre texte et image sont éminemment variables : une parole inscrite dans le champ d'une scène de victoire, un nom identifiant un personnage ou la référence au magistrat-émetteur ne relèvent pas de la même intention et jouent naturellement des rôles différents. La fonction du texte, son insertion dans le champ de l'image, les jeux qu'il autorise sont toutefois des éléments pris en compte dans les analyses des différents intervenants et pourraient être approfondis.

Les répertoires céramiques étudiés par trois intervenants fournissent peu d'exemples de textes inscrits; V.Zachari et G. Cogan en signalent néanmoins chacune de rares exemples. Sur une amphore du peintre de Nikon, conservée à Boston ${ }^{10}$ et citée par V. Zachari, apparaissent ainsi deux inscriptions exaltant la beauté (kalos); mais l'une, associée à un pilier hermaïque, est explicite et vante les charmes d'un jeune homme, Glaukos, tandis que l'autre, inscrite au-dessus de l'autel, semble renvoyer à une idée plus désincarnée de la beauté, celle qui plaît aux dieux à l'instar de la fumée qui monte des autels. Ces choix sont d'autant plus significatifs que les scènes retenues pour l'analyse sont dépourvues de présence humaine. Un jeu analogue se retrouve sur une hydrie du British Museum ${ }^{11}$ analysée par G. Cogan : l'inscription " Nike kale » renvoie autant à la beauté de la déesse qu'à celle de l'exploit athlétique. Dans ces deux cas, les textes relèvent d'une pratique bien identifiée et relativement bien attestée, celle des inscriptions acclamatoires ; loin d'être simplement anecdotiques, ces inscriptions acquièrent par leur insertion dans le champ de l'image une puissance suggestive nouvelle, révélant un aspect original des solutions figuratives mises en œuvre dans la céramique attique ${ }^{12}$.

Si les légendes des revers monétaires ne sont pas explicitement au centre de l'étude de Y. Berthelet, elles sont en réalité essentielles au raisonnement, dans la mesure où elles précisent l'identité du magistrat-émetteur, auquel les symboles sacerdotaux représentés renvoient, directement ou indirectement. Les contraintes propres au support numismatique (espace réduit, conventions régissant l'expression de l'autorité émettrice) ne permettent guère de développer le texte écrit; dans le contexte crucial des luttes de la fin de la République romaine, le recours aux symboles sacerdotaux s'inscrit dans le développement foisonnant des représentations figurées et la mise en place d'un discours figuratif particulièrement riche ${ }^{13}$. Texte et image se complètent alors, dans un système de représentations sans cesse complexifié, qui réflète l'intensité de la compétition politique.

$\mathrm{Au}$ final, les différentes interventions confirment tout l'intérêt d'une histoire par l'image, au prix de la constitution d'outils adaptés, d'une rigueur méthodologique sans cesse renouvelée et d'une contextualisation précise. C'est à ces conditions que les jeunes 
chercheurs pourront révéler les mécanismes figuratifs propres à chaque support, à chaque culture, au cœur de notre propre imaginaire, et s'en servir pour penser les différents systèmes de représentations.

\section{BIBLIOGRAPHIE}

ALFöLDI A. (1956), « The Main Aspects of Political Propaganda on the Coinage of the Roman Republic », dans Essays in Roman Coinage Presented to Harold Mattingly, Oxford, p. 63-95.

BEAZLEY J. (1956), Attic Black-Figure Vase Painters, Oxford.

BEAZLEY J. (1963), Attic Red-Figure Vase Painter², Oxford.

DENOYELLE M. et IOZZO M. (2009), La céramique grecque d'Italie méridionale et de Sicile, Paris.

CRAWFORD M. H. (1974), Roman Republican Coinage, Cambridge.

EKROTH G. (2009), « Why (not) paint an Altar? A study of Where, When and Why Altars appear on Attic-Red-figure Vases », dans Nørskov V. et al. éd., The World of Greek Vases, Rome, p. 89-114.

JAILLARD D. (2001), « Le pilier hermaïque dans l'espace sacrificiel », MEFRA 113, p. 341-363.

LISSARRAGUE F. (1985), « Paroles d'images : remarques sur le fonctionnement de l'écriture dans l'imagerie attique », dans Christin A.-M. éd., Écritures II, Paris, p. 71-93.

LISSARRAGUE F. (1992), « Graphein : écrire et dessiner », dans Bron Ch. et Kassapoglou E. éd., L'image en jeu, Lausanne, p. 189-203.

LISSARRAGUE F. (2006), « De l'image au signe. Objets en représentation dans l'imagerie grecque », Les Cahiers du CRH 37, p. 11-24.

MORET J.-M. (1984), « Le jugement de Pâris en Grande-Grèce : mythe et actualité politique », Antike Kunst 21, p. 76-98.

PEREZ C. (1986), Monnaie du pouvoir. Pouvoir de la monnaie. Une pratique discursive originale: le discours figuratif monétaire ( ${ }^{\text {er }}$ s. av. J.-C $-I V^{e}$ ap. J.-C.), Paris.

RÜPKE J. (2005), Fasti sacerdotum. Die Mitglieder der Priesterschaften und das sakrale Funktionspersonal römischer, griechischer, orientalischer und jüdisch-christlicher Kulte in der Stadt Rom von 300 v. Chr. Teil 1 :Jahres- und Kollegienlisten. Teil 2: Biographien. Teil $3:$ Quellenkunde und Organisationsgeschichte, Stuttgart-Munich.

SIEBERT A.-V. (1999), Instrumenta sacra. Untersuchungen zu römischen Opfer-, Kult- und Priestergeräten, Berlin-New York.

TRENDALL A. D. (1967-1983), The Red-figured Vases of Lucania, Campania and Sicily, Oxford.

TRENDALl A. D. et CAmbitoglou A. (1978-1992), The Red-figured Vases of Apulia, Oxford-Londres. 


\section{NOTES}

1. Cf. les résultats de la vaste entreprise du Corpus Vasorum Antiquorum consultables sur le site du Beazley Archiv de l'université d'Oxford. URL : http://www.beazley.ox.ac.uk/cva/

2. B EAZLEY 1956; BeAZLEY 1963. Base de données en ligne (abréviation: BA). URL: http:// www.beazley.ox.ac.uk/index.htm

3. ЕКROTH 2009 ; JAILLARD 2001.

4. TRendall 1967-1983; TRANDAll et Cambitoglou 1978-1992; Denoyelle et Iozzo 2009.

5. CRAWFORD 1974.

6. RÜPKE 2005 .

7. SIEBERT 1999.

8. MORET 1984.

9. Voir les analyses lumineuses de LISSARRAGUE 2006.

10. BA : 275790 .

11. BA : 11818 .

12. Cf. les travaux de François Lissarrague, en particulier LISSARRAGUE 1985 ; LISSARRAGUE 1992.

13. Voir par exemple ALFÖLDI 1956 ; PÉREZ 1986.

\section{AUTEUR}

\section{SYLVIA ESTIENNE}

École Normale Supérieure-Paris ; ANHIMA - UMR 8210 